\title{
Special issue of biomedical engineering letters on advances in intelligent prostheses
}

\author{
Liming Salvino ${ }^{1}$. Wing Kong Chiü ${ }^{2}$ Jerome Lynch ${ }^{3} \cdot$ Kenneth J. Loh ${ }^{4}$
}

Published online: 18 February 2020

(c) Korean Society of Medical and Biological Engineering 2020

\section{Preface}

This Special Issue comprises original and review papers from engineering and medical professionals expounding on the state of the art in engineering and the physical sciences that aim to enhance the performance of prosthetic devices including socket prostheses and osseointegrated prostheses.

Advances in physical science disciplines are poised to revolutionize how doctors and patients view and use prostheses. With monitoring and control capabilities, advanced prostheses can provide functionalities far beyond simple limb extension and load support. Prostheses with built-in sensing, embedded intelligence such as real-time monitoring and infection eradication, self-actuation, and control have been quickly advancing over the past 10 years or so. Given the rapid emergence of these transformative developments, this Special Collection is designed to collate a volume of work devoted to cutting-edge developments in advances in intelligent prostheses that enhance medical care and the quality of life of patients with limb loss. Additionally, these prosthetic functionalities and technologies may be utilized as "special wearables" to collect and mine human physiological data in order to gain knowledge in fundamental sciences

Wing Kong Chiu

Wing.kong.chiu@monash.edu

Liming Salvino

liming.salvino@gmail.com

Jerome Lynch

jerlynch@umich.edu

Kenneth J. Loh

kenloh@ucsd.edu

Office of Naval Research, Arlington, VA 22203, USA

Monash University School of Engineering, Clayton, Australia

3 University of Michigan, Ann Arbor, USA

4 University of California San Diego, La Jolla, CA, USA on neurological and biomechanical effects of injury and rehabilitation.

This Special Collection consists of eight papers on the topic of "Advances in Intelligent Prostheses." The following is a brief summary of the science and technology focus of each paper:

I. "The State of the Art of Osseointegration"-Osseointegration (OI) technology involves the direct attachment of bone onto a titanium implant allowing direct connections to an external prosthesis. OI patients demonstrate encouraging improvements in quality of life and function. This review paper provides an OI technology overview and discusses the pros and cons of different types of implants. It also provides examples of clinical applications, current development efforts, challenges, and future opportunities [1].

II. The original paper on "Quantitative Assessment of Compress-Type Osseointegrated Prosthetic Implants in Human Bone using Electromechanical Impedance Spectroscopic Methods" describes the development of sensing strategies that can be deployed on the percutaneous end of osseointegrated prostheses to clinically measure the degree of osseointegration at the bone-fixture interface using guided wave methods. A brief overview of guided waves for assessing osseointegration is first presented. Then the paper describes a new interrogation strategy tailored for compress-type fixtures using electromechanical impedance measurements of piezoelectric elements bonded to prosthesis fixture [2].

III. In order for the OI prosthesis to be successful, it must achieve and remain free of infection. The review paper "Electrochemical Methods to Enhance Osseointegrated Prostheses" focuses on the electrochemical methods that may be utilized to modify and/or monitor the interfacial microenvironment in which the metallic implant interacts with the biological sys- 
tem (host bone tissue or microbes). These methods described in this review are also directly applicable to other medical devices, such as total joint replacements and dental implants [3].

IV. "Advancements in Functional Materials for Potential as Wearable Infection Sensors in Limb Prostheses" introduces the integration of infection sensors to prostheses for detecting and preventing infections for both traditional socket and OI prostheses. Since pathogenic volatiles have been identified as potent stimuli, this paper reviews the current techniques in the field of infection sensing, specifically focusing on identifying portable and flexible sensors with the potential to be integrated into prosthesis designs [4].

V. The paper on "Non-Radiative Healing Assessment Techniques for Fractured Long Bones and Osseointegrated Implant" provides an overview of the fracture healing process of long bones, a review of work that proposed appropriate physical parameters for the assessment of healing and highlights some recent work that reported on the development of nonradiative techniques for healing assessment. It also includes an overview of the development and monitoring of osseointegration for trans-femoral osseointegrated implant. This paper describes non-radiative techniques that are useful for healing assessment and the stability assessment of osseointegrated implants. The achievement of non-radiative quantitative assessment methodologies to determine the state of healing of fractured long bones and to assess the stability of osseointegrated implant will shorten the patient's rehabilitation time, allowing earlier mobility and return to normal activities [5].

VI. "A Review on the Latest Advancements in the Noninvasive Evaluation/Monitoring of Dental and Transfemoral Implants"- this paper provides a comprehensive review of clinically-approved and emerging noninvasive methods to evaluate/monitor the osseointegration of dental and orthopaedic prostheses. A discussion about the advantages and limitations of each method is provided based on the outcomes of the cases presented. The review of the emerging technologies covers the developments of the last decade, while the discussion about the clinically approved systems focuses mostly on the latest (2017-2018) findings [6].

VII. "Sensing and Actuation Technologies for Smart Socket Prostheses" provides a detailed review of various sensing and actuation technologies that have been proposed and integrated for realizing higher performance smart socket prostheses. In particular, the review paper emphasizes how different sensing technologies have been used to measure the three critical factors that affect socket and amputee comfort, which include contact pressure distribution, local temperature, and volume fluctuations of the residual limb. Furthermore, sensors that have been employed for monitoring amputee gait patterns, as well as infection in the residual limb due to socket-induced skin issues, are also highlighted. On the other hand, various actuation technologies recommended for enhancing socket performance are also discussed [7].

VIII. The goal of the review paper "Advanced Technologies for Intuitive Control and Sensation of Prosthetics" is to examine the state-of-the-science in the areas of intuitive control and sensation of prosthetic devices. Recent research has made significant advancement not only in the intuitive use of electromyography for control but also in the ability to provide relevant meaningful perceptions through various stimulation approaches. While much of this previous work has traditionally focused on those with upper extremity amputation, new developments include advanced bidirectional neuroprostheses that are applicable to both upper and lower limb amputation. Current research and development efforts in external systems, implanted systems, surgical approaches, and regenerative approaches are also discussed [8].

As stated previously, papers in this Special Issue of Biomedical Engineering Letters on Advances in Intelligent Prostheses cover a wide range of technological topics in engineering, physical sciences, and medicine. We hope that this volume will promote further communication and collaboration involving cross-disciplinary communities toward the goals of key technology advancement for intelligent prostheses and for the adoption and further development of the technology for monitoring the health, fitness, and performance of humans in athletic, medical, and military applications. We would like to thank all the participating authors for their invaluable contributions. A special thanks to BMEL's editor-in-chief, Professor Jae Sung Lee, for his patience, support, and encouragement in putting together this Special Issue.

\section{Compliance with ethical standards}

Conflict of interest The author declares that there is no conflict of interest regarding the publication of this paper.

Ethical approval This article does not contain any studies with human participants or animals performed by any of the authors. 


\section{References}

1. Overmann AL, Forsberg JA. The state of the art of osseointegration for limb prosthesis. Biomed Eng Lett. 2019. https://doi. org/10.1007/s13534-019-00133-9.

2. Wang W, Lynch JP. Quantitative assessment of compress-type osseointegrated prosthetic implants in human bone using electromechanical impedance spectroscopic methods. Biomed Eng Lett. 2019. https://doi.org/10.1007/s13534-019-00139-3.

3. Ehrensberger MT, Clark CM, Canty MK, McDermott EP. Electrochemical methods to enhance osseointegrated prostheses. Biomed Eng Lett. 2019. https://doi.org/10.1007/s13534-019-00134-8.

4. Devaraj H, Aw KC, McDaid AJ. Review of functional materials for potential use as wearable infection sensors in limb prostheses. Biomed Eng Lett. 2019. https://doi.org/10.1007/s13534-01900132-w.

5. Lu S, Vien BS, Russ M, Fitzgerald M, Chiu WK. Non-radiative healing assessment techniques for fractured long bones and osseointegrated implant. Biomed Eng Lett. 2019. https://doi. org/10.1007/s13534-019-00120-0.

6. Rizzo P. A review on the latest advancements in the non-invasive evaluation/monitoring of dental and trans-femoral implants. Biomed Eng Lett. 2019. https://doi.org/10.1007/s13534-01900126-8.

7. Gupta S, Loh KJ, Pedtke A. Sensing and actuation technologies for smart socket prostheses. Biomed Eng Lett. 2019. https://doi. org/10.1007/s13534-019-00137-5.

8. Wolf EJ, Cruz TH, Emondi AA, Langhals NB, Naufel S, Peng GCY, Schulz BW, Wolfson M. Advanced technologies for intuitive control and sensation of prosthetics. Biomed Eng Lett. 2019. https://doi.org/10.1007/s13534-019-00127-7.

Publisher's Note Springer Nature remains neutral with regard to jurisdictional claims in published maps and institutional affiliations. 